Hydroécol. Appl. (1992) Tome 4 Vol 2, pp. 105-121

\title{
Modélisation de l'écosystème pélagique en Manche: du modèle global de la Manche au modèle côtier du littoral nord-breton
}

\author{
J. Rietjens*, A. Agoumi ${ }^{\star *}$,
}

- EDF, Direction des Études et Recherches, 6, Quai Watier, 78400 Chatou, France.

- École Hassania des Travaux Publics, BP 8108 Oasis Casablanca, Maroc.

Résumé. - Dans une première étape, un modèle global a été développé à l'échelle de la Manche entière. Ce modèle bicouche permet de représenter, sur des cycles pluriannuels, l'évolution de la température, de la biomasse phytoplanctonique et zooplanctonique et de l'azote sous différentes formes, dans les couches de surface et de fond. Ce modèle a en particulier montré que le comportement écologique du système Manche était très lié à la part respective de deux facteurs hydrodynamiques majeurs: les frottements des courants sur le fond et le cisaillement dû au vent en surface. A l'Est du chenal, le frottement au fond étant trop important, aucune stratification n'est possible; à l'Ouest, la stratification existe, son ampleur dépendant de l'importance relative des deux facteurs. L'application, dans une seconde phase, de ce modèle à la zone côtière nord-bretonne a confirmé la bonne adéquation à l'échelle régionale des mécanismes hydrodynamiques considérés dans le modèle global. Elle a par contre montré que, du point de vue biologique, le schéma adopté ne suttisait pas à décrire l'écosystème phytoplanctonique côtier. L'introduction des apports azotés d'origine fluviale et du compartiment benthique algal dans le modèle côtier a permis de simuler un comportement du système cohérent avec les observations in situ. On a montré notamment que la végétation fixée limite l'effet potentiel des rejets azotés d'origine fluviale sur le plytoplancton.

A l'issue de cette étude générale sur la Manche, on dispose d'outils numériques permettant de représenter l'évolution de l'écosystème marin et de tester l'impact de certains agents polluants. II apparaît que, si les mécanismes hydrodynamiques régissant le système sont identiques à l'échelle globale ou locale, les facteurs biologiques essentiels dans l'équilibre de l'écosystème différent d'une échelle à l'autre: ainsi la pollution d'origine agricole s'avère être perturbatrice à l'échelle côtière alors que son impact, à l'échelle de la Manche entière, est aujourd'hui négligeable.

Abstract. - During the first project phase, a global scale model for the entire English Channel was developed. With this two-layer model, we can represent changes over pluri-annuel cycles in temperature, phytoplankton and zooplankton biomass, and nitrogen 
in various forms, in the surface and bottom layers. The model most notably demonstrated that the ecological behavior of the Channel system is closely linked to the respective impact of two major hydrodynamic factors: friction of currents on the bottom layer and shear due to wind at the surface. In the eastern part of the Channel, no stratification is possible, as friction is too great on the bottom; in the west, stratification does exist to varying degrees depending on the relative impact of the two factors.

In the second phase, application of this model to the northern Breton coastal zone confirmed that the hydrodynamic mechanisms considered in the global model were well adapted on a regional scale. On the other hand, it indicated that, from a biological point of view, the representation adopted did not adequately describe the coastal phytoplankton ecosystem. Introduction into the coastal model of fluvial nitrogen inflow and benthic algal vegetation enabled simulation of system behavior consistent with the field observations. In particular, it was demonstrated that benthic vegetation limits the potential impact on phytoplankton of fluvial nitrogen inflow.

At the conclusion of this general study of the Channel, we now have numerical models for representation of evolution in the marine ecosystem and for testing of the impact of certain pollutants. It appears that, while the hydrodynamic mechanisms governing the system are identical on the global and regional scales, the biological factors essential to the balance of the ecosystem differ from one scale to the other.

\section{INTRODUCTION}

Le développement industriel et agricole le long du littoral de la Manche a atteint dans les années 80 un seuil important. Différents signes d'alarme sont venus indiquer que le niveau de pollution auquel la Manche a été soumise pendant longtemps était devenu inacceptable pour l'équilibre écologique côtier: eaux rouges, espèces toxiques, prolifération excessive d'algues...

Electricité de France, cherchant à suivre et à cerner l'impact sur l'écosystème Manche, de ses aménagements le long du littoral, a lancé en 1980 , un projet de recherche visant une meilleure compréhension de la dynamique physique, et biologique de cette mer.

Dans une première phase de ce projet, un intérêt particulier a été porté aux mécanismes essentiels qui conditionnent l'évolution saisonnière thermique et biologique des eaux de la Manche à grande échelle spatiale: une modélisation de la structure thermique et planctonique a été faite sur un maillage couvrant toute la Manche avec un pas d'espace de 6 milles marins. Ce modèle est dit Modèle global de la Manche.

Dans une seconde phase, un modèle côtier du littoral nord-breton dit Modèle côtier a été élaboré. L'objectif de ce modèle, plus précis, a été de cerner l'impact des rejets côtiers de cette zone sur l'équilibre du système.

\section{MODĖLE GLOBAL DE LA MANCHE}

Le modèle développé est particulièrement axé sur l'évolution thermique et 
sur la dynamique phytoplanctonique de la Manche. II a été élaboré en deux étapes; un modèle simulant le régime thermique de la Manche a d'abord été développé. Ce modèle intégrant les processus hydrodynamiques a été conçu de façon à pouvoir réaliser des simulations sur de longues périodes (plusieurs années), ce qui est nécessaire dans la plupart des études biologiques et sur une zone aussi étendue que la Manche. Dans une seconde étape, un modèle de production primaire a été établi et associé au modèle thermique afin d'étudier les grandes tendances de l'évolution saisonnière phytoplanctonique et leur dépendance des conditions physiques de ce milieu.

\section{Modélisation du régime thermique}

Le modèle thermique développé permet de reconstituer l'évolution des températures de surface et de fond en tout point de la Manche tout au long des différents cycles annuels, en utilisant des données de courant résultant de modèles mathématiques Warluzel, et Manoha, 1977) et des données météorologiques relevées à la station de La Hague (Agoumi, 1982), (Agoumi et al., 1983).

Verticalement, ce modèle présente une structure à deux couches homogènes d'épaisseurs variables (fig. 1):

- la couche de surface est soumise au flux de chaleur dû aux échanges air-mer et à l'action du vent;
- la couche de fond est soumise aux frottements des courants marins sur le fond.

Horizontalement, il est bidimensionnel, couvrant, avec un pas d'espace de 6 milles marins, une zone s'étendant en longitude de $6^{\circ}, 5 \mathrm{~W}$ à $2^{\circ} \mathrm{E}$, de $48^{\circ} \mathrm{N}$ à $51^{\circ} \mathrm{N}$ en latitude (fig.2). II prend en compte les échanges dispersifs liés aux courants de marée et le transport résiduel induit conjointement par le vent et les marées (représentés respectivement dans le modèle par un terme diffusif et un terme advectif).

II calcule, avec un pas de temps de 3 heures, la température et l'épaisseur des deux couches dans chaque maille. Les résultats ont été comparés aux données de température de surface disponibles pour la période de simulation 1960-1979. La comparaison apparaît réaliste (fig. 3,4 ).

Ce modèle a aussi permis de reproduire la formation et l'évolution annuelle de la zone frontale entre les eaux stratifiées de l'ouest et les eaux homogènes de l'est de la Manche (fig. 5).

L'analyse des résultats obtenus montre que l'effet des échanges horizontaux, en particulier celui de la dérive, est assez limité sur le régime thermique; celui-ci semble être plus conditionné par les processus locaux (échanges air-mer et structure hydrodynamique verticale). 

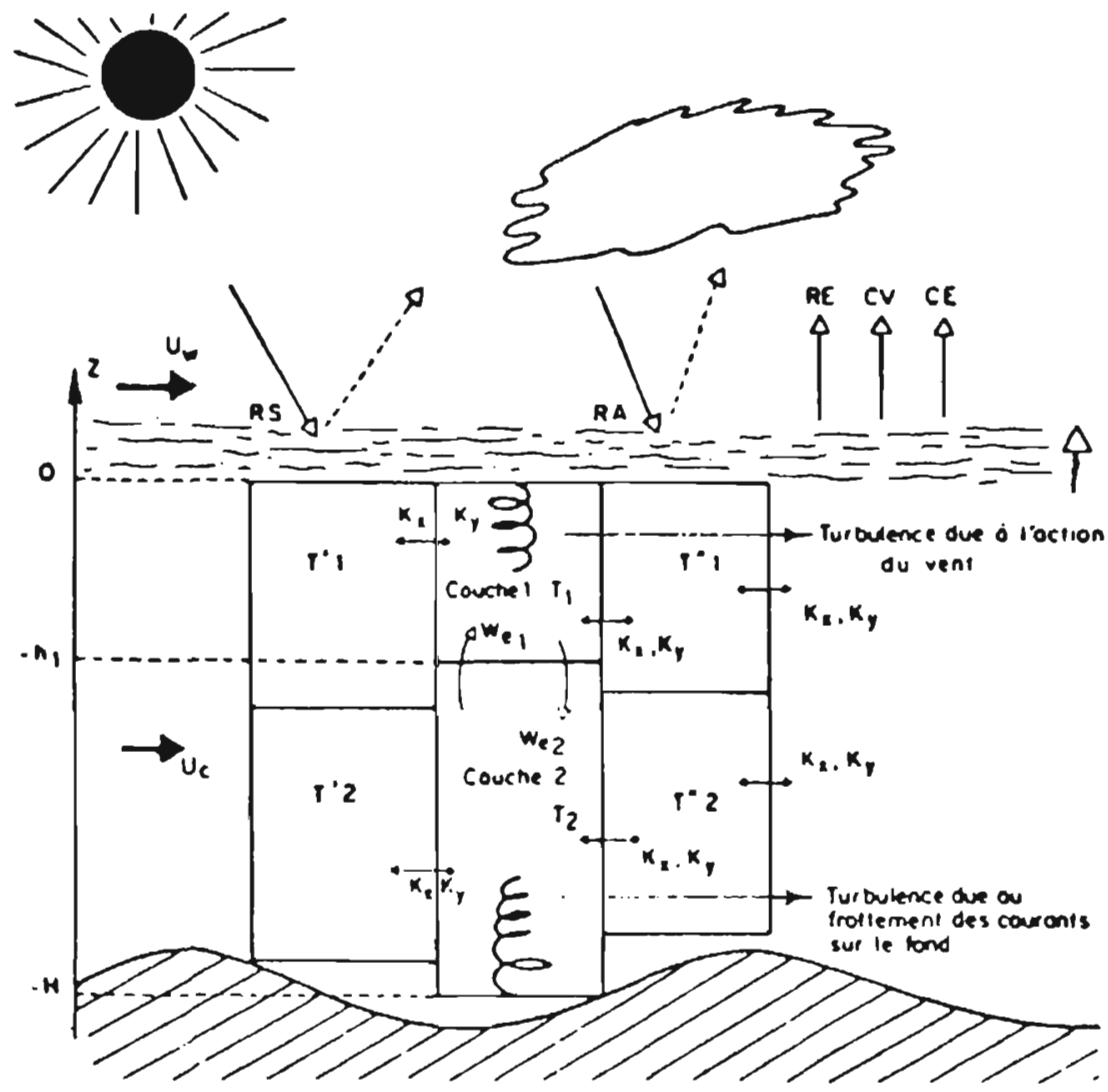

Fig. 1. Structure du modèle thermique.

$W_{1}$ : Vilesse d'entrainement des masses d'eau de la couche 2 à la couche 1.

$\mathrm{We}_{2}$ : Vitesse d'entrainement des masses d'eau de la couche 1 à la couche 2.

$U_{c} \quad$ : Vitesse horizontale des masses d'eau.

$U_{w} \quad$ : Vitesse du vent.

$\mathrm{Kx}, \mathrm{Ky}$ : Coefficients de dispersion.

CE : Flux de chaleur dû à l'évaporation.

CV : Flux de chaleur dû à la convection.

RE : Rayonnement de l'eau.

RS : Rayonnement solaire.

RA : Rayonnement atmosphérique. 


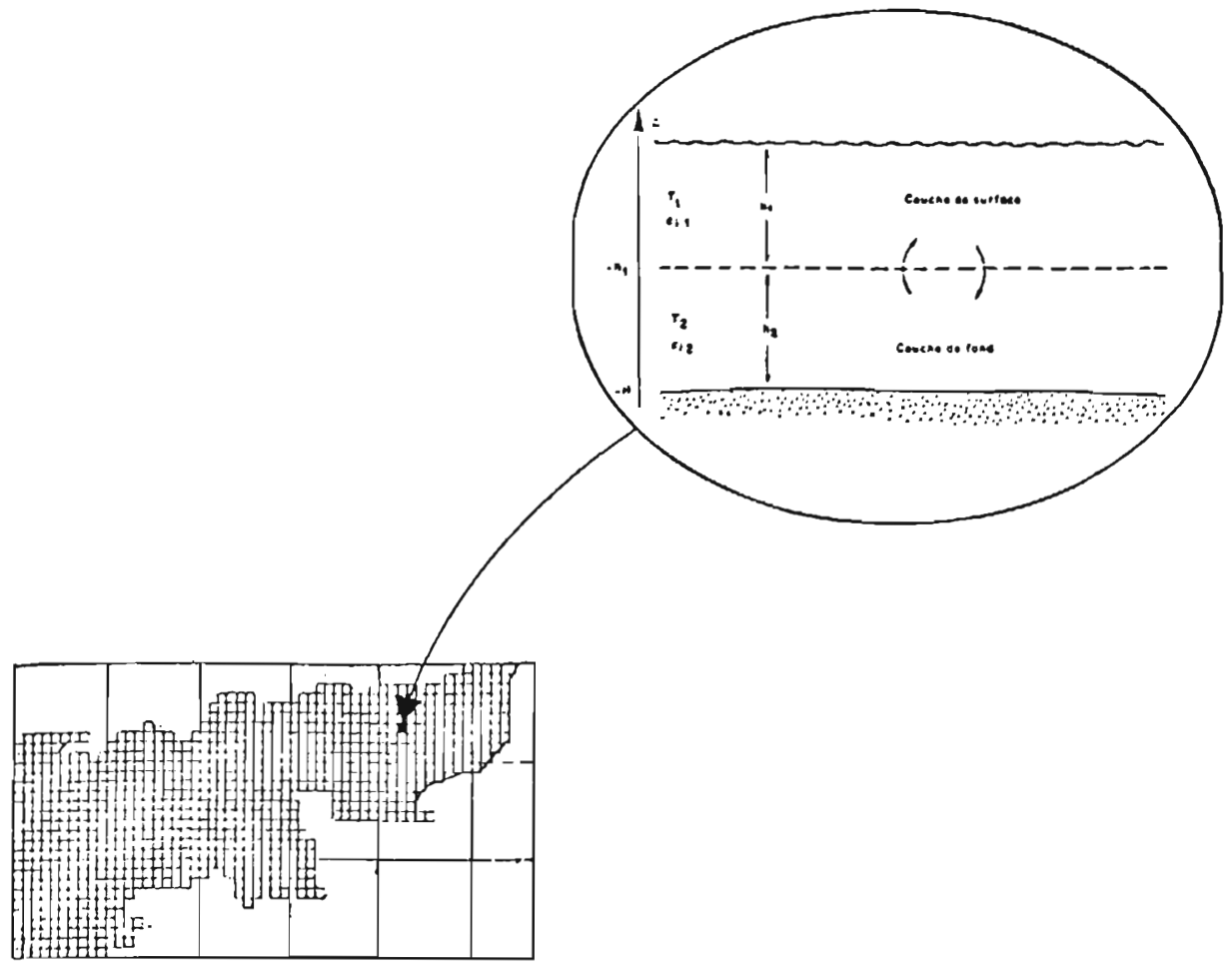

Fig. 2. Maillage considéré dans la simulation et structure verticale dans chaque maille.

\section{Modélisation de l'évolution saisonnière du système planctonique}

Un modèle de production primaire a été développé. II permet de simuler l'évolution saisonnière des concentrations en phytoplancton $\left(\mathrm{mg} \mathrm{chla} / \mathrm{m}^{3}\right)$, en zooplancton herbivore $\left(\mathrm{mg} \mathrm{C} / \mathrm{m}^{3}\right)$, en azote nitrique $(\mu \operatorname{atg} N / I)$, en azote ammoniacal ( $\mu$ atg $N / I)$, en azote organique détritique d'origine zooplanctonique et en azote organique détritique d'origine non zooplanctonique ( $\mu$ atg $N / /$ ) (fig. 6). Les intéractions chimiques et biologiques retenues entre ces différentes variables résultent de différents travaux publiés dans la littérature (Agoumi et al., 1985).

Un couplage entre le modèle du régime thermique et ce modèle de production primaire a été réalisé. Le modèle résultant permet de calculer, en chaque point du maillage considéré $(6$ milles $\times 6$ milles), l'évolution saisonnière en surface et au fond de la 

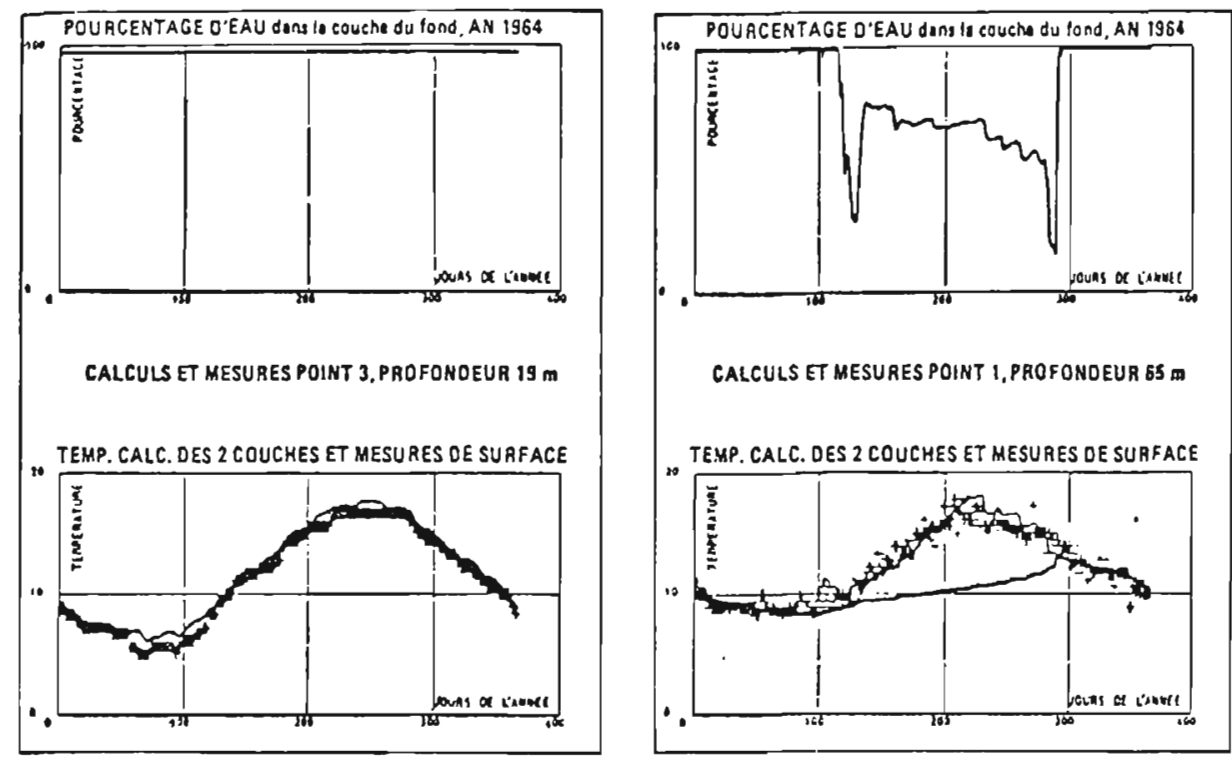

Fig. 3. Températures calculées et mesurées au point 3, en Manche orientale, pour l'année 1964. Fig. 4. Calculs et mesures au point 1. Manche orientale.

température, des concentrations en phytoplancton, en zooplancton et en azote sous différentes formes.

Les résultats des différentes simulations faites avec ce modèle ont permis de mettre en évidence certaines caractéristiques propres à ce système (fig. 7, 8, 9).

1. Une différence de comportement importante du système planctonique dans la partie ouest et la partie est de la Manche, ceci étant lié à la présence de fortes stratifications à l'ouest et à la présence d'une structure pratiquement homogène à l'est.

2. Même en Manche occidentale, on retrouve une différence de comportement entre le large des côtes fran- çaises et des côtes anglaises. Elle s'explique par la nature des structures hydrologiques locales.

3. Les zones de transition séparant les eaux stratifiées de l'ouest et les eaux homogènes de l'est de la Manche connaissent une production planctonique particulièrement importante.

4. En Manche orientale, l'effet des apports nutritifs de la Seine reste assez limité géographiquement, il concerne essentiellement l'est de la baie de Seine et la côte du Pays de Caux.

Une analyse de sensibilité de ce modèle aux échanges hydrodynamiques horizontaux, à la turbidité des eaux, à leur composition en azote et 


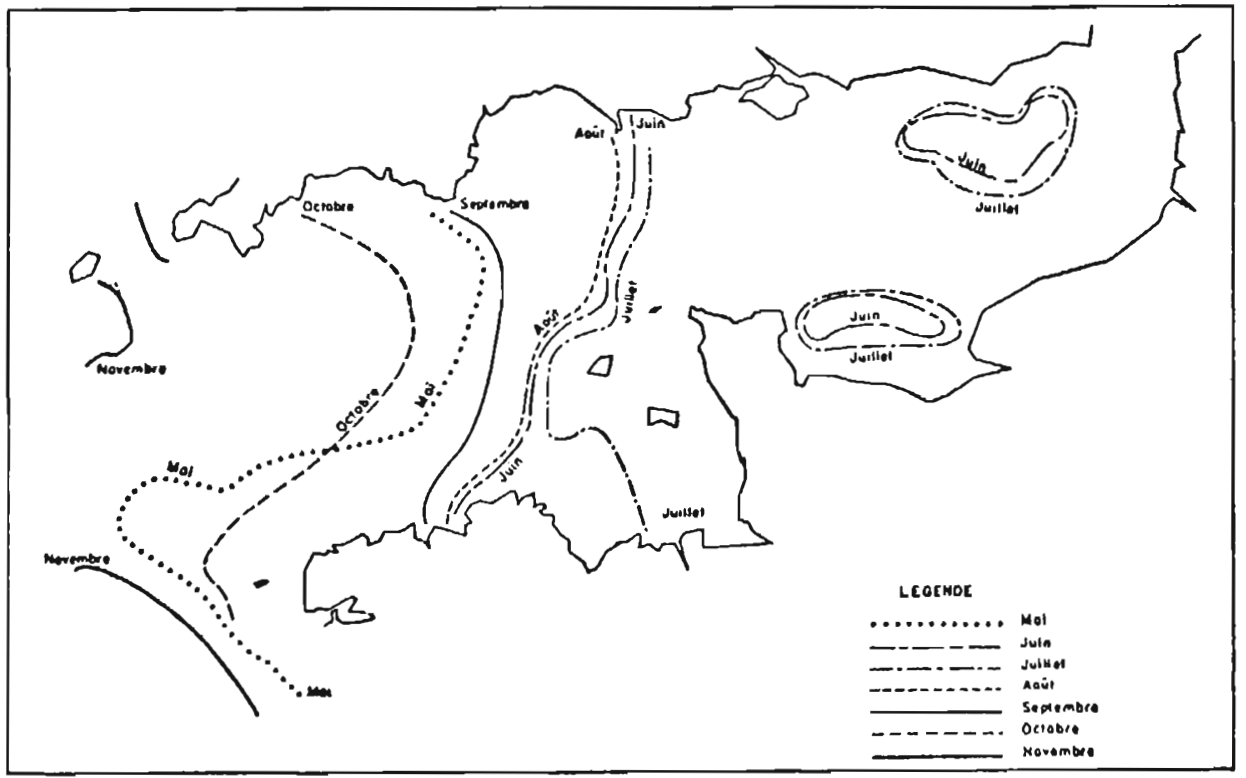

Fig. 5. Déplacement tout au long de l'année des lignes d'écart des températures surface-fond égaux à $0,5^{\circ} \mathrm{C}$.

aux variations climatiques a été effectuée: il apparaît que les grandes tendances de l'évolution saisonnière du système phytoplanctonique, pour une évolution météorologique et un niveau de nutriments donnés, sont d'abord liées aux conditions hydrodynamiques locales et à la turbidité des eaux. Les échanges horizontaux, l'hétérogénéité horizontale en azote... paraissent comme des facteurs secondaires dont le rôle est plutôt d'accentuer ou de modérer les gradients horizontaux en chlorophylle a induits par les premiers. Ce résultat va dans le même sens que celui obtenu lors de l'étude thermique; ce sont d'abord les phé- nomènes locaux qui conditionnent l'évolution du système.

Le facteur turbidité prend une très grande importance à l'est de la Manche où son domaine de variation spatio-temporelle est grand. Les résultats du modèle dans cette région en sont tout à fait dépendants: ceci a été conforté lors d'une confrontation entre les calculs et les mesures relevées en avril et juin 1984 lors des campagnes de mesures Plenum que nous avons réalisées en Manche orientale (Agoumi, 1985).

En Manche occidentale, les variations spatio-temporelles de la turbidité 


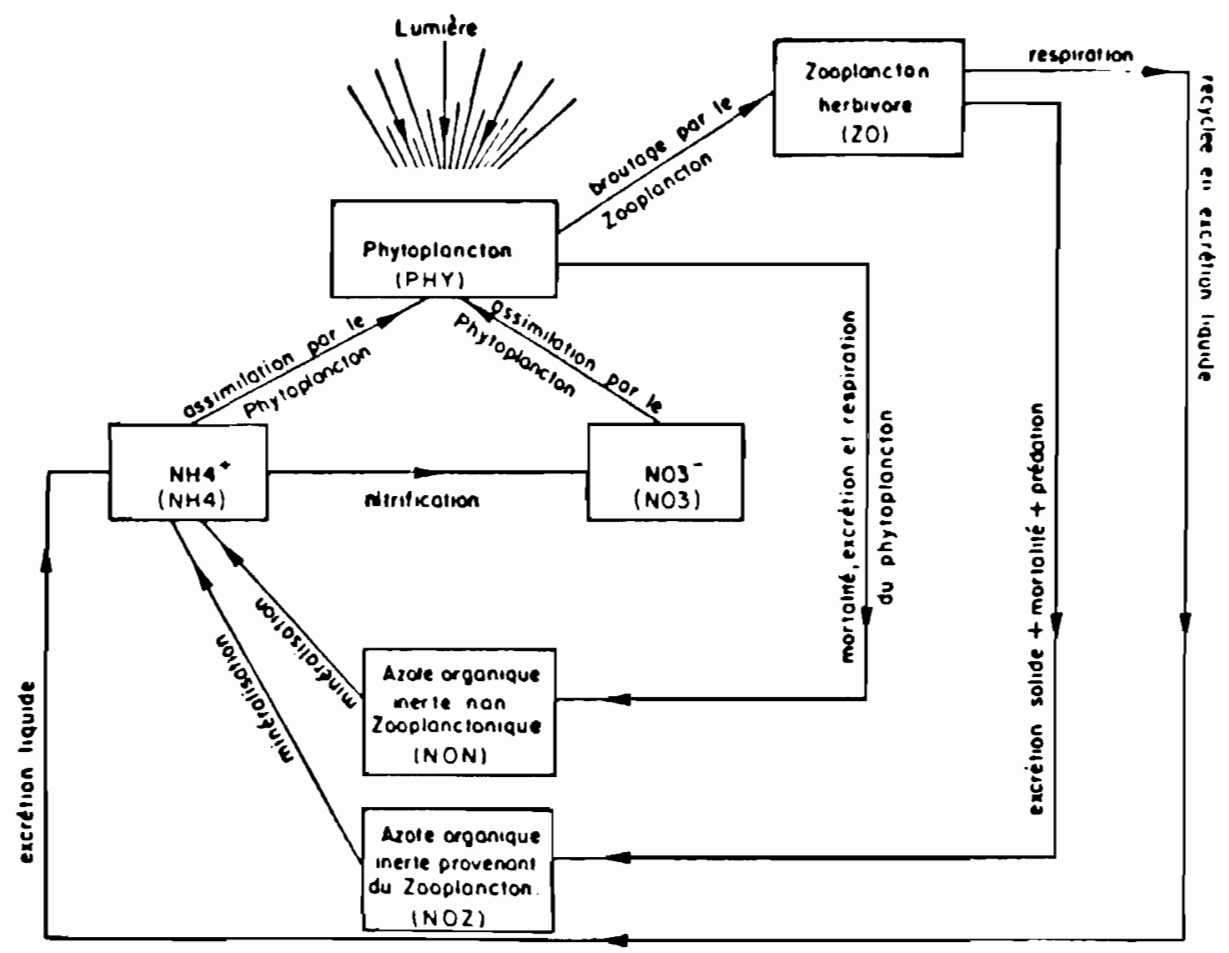

Fig. 6. Représentation schématique de l'écosystème modélisé.

sont faibles; ce sont plutôt les conditions hydrodynamiques locales qui déterminent l'évolution saisonnière du système phytoplanctonique.

\section{MODĖLE D'ÉCOSYSTĖME CÔTIER}

\section{Étude de la zone côtière nord-bretonne}

Le modèle précédemment développé à l'échelle de la Manche ayant montré la bonne adéquation des mécanismes simulés à la représentation de l'écosystème phytoplanctonique, il est apparu logique de transporter le schéma adopté (bidimensionnel horizontal à deux couches homogènes verticales) au littoral Nord-Breton. Néanmoins, le modèle a été adapté pour:

- s'appliquer à un maillage plus fin (mailles de $2 \mathrm{~km} \times 2 \mathrm{~km}$ au lieu de $10 \mathrm{~km}$ et pas de temps de 1 heure, fig. 10), en utilisant les données bathymétriques et courantologiques fournies par le Laboratoire National d'Hydraulique (Regimbaud, 1987), 
Surface
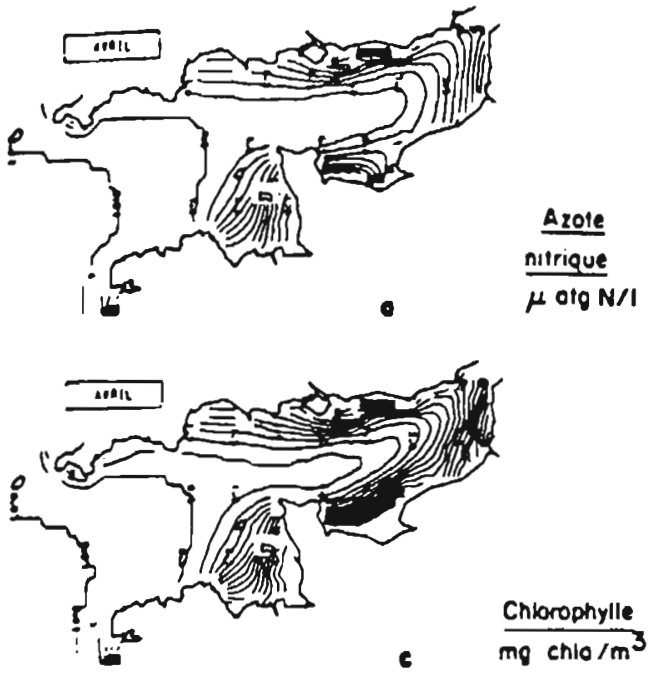

Iond
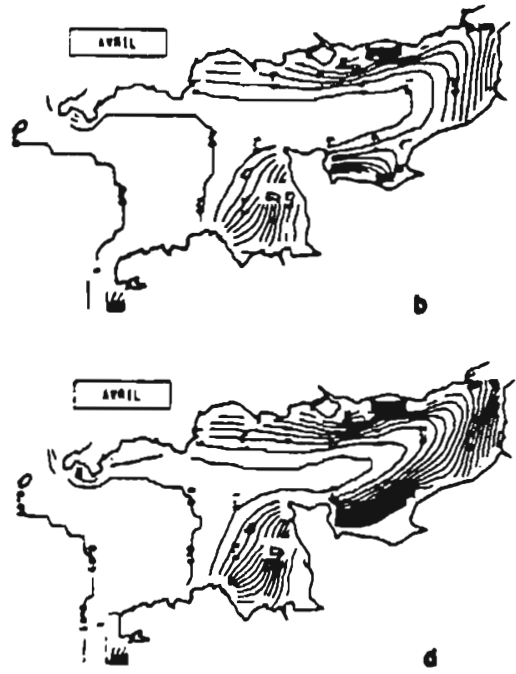

Fig. 7. Résultats de simulation - 1976 (avril).

(a,b) Concentration en Azote nitrique en surface (et au fond) $\mu$ atg $N /$

Surfoce

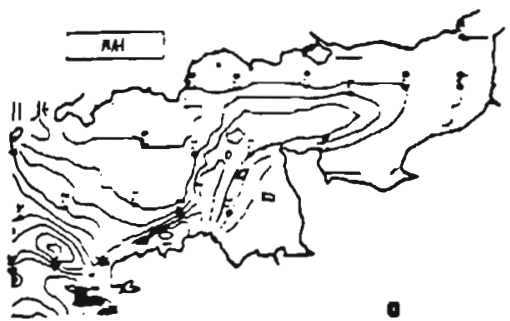

Azote

nitrique $\mu \operatorname{olg} N / 1$

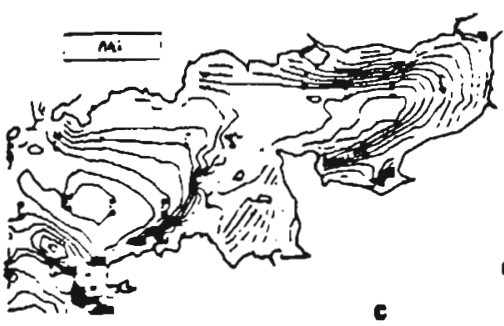

lond

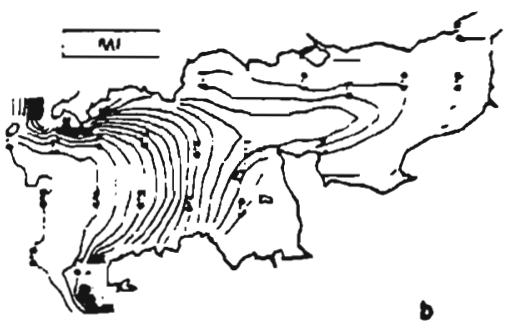

Chiorophylle

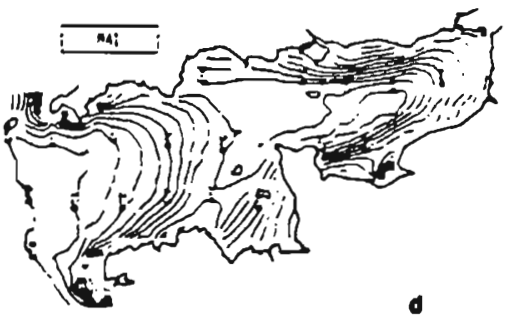

Fig. 8. Résultats de simulation - 1976 (mai).

(c, d) Concentration en Chlorophylle a en surface (et au fond) $\mathrm{mg} \mathrm{Chla} / \mathrm{m}^{3}$. 

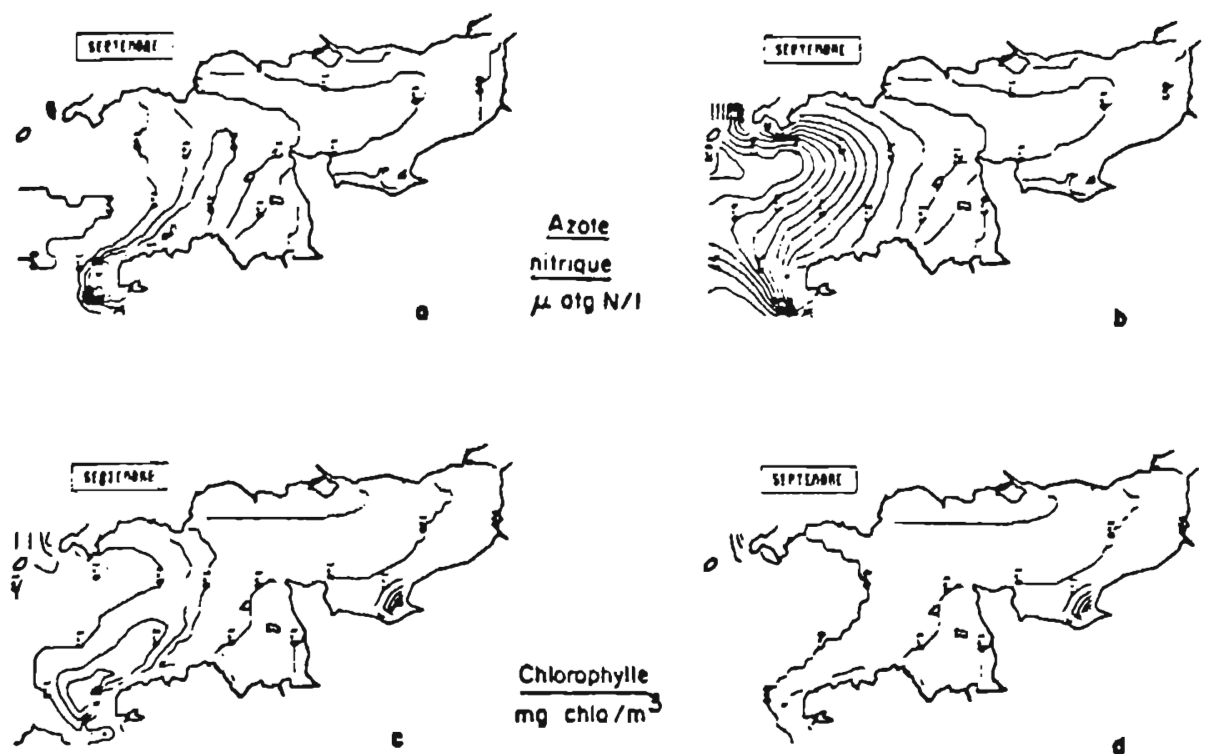

$\frac{\text { Chlorophylle }}{\mathrm{mg} \text { chlo } / \mathrm{m}^{3}}$

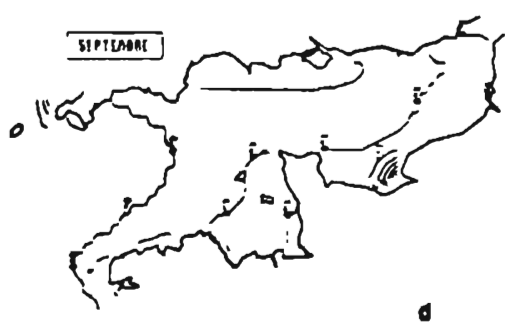

Fig. 9. Résultats de simulation - 1976 (septembre)

$(a, b)$ Concentration en Azote nitrique en surface (et au fond) $\mu$ atg $N / 1$

(c, d) Concentration en Chlorophylle a en surface (et au fond) $\mathrm{mg} \mathrm{Chla} / \mathrm{m}^{3}$.

cette nouvelle échelle spatiale et temporelle nécessitant un recalage des coefficients de diffusion décrivant l'effet dispersif des marées,

- utiliser les résultats de simulation du modèle global comme conditions aux limites en mer ouverte, selon la théorie des modèles emboîtés (contrairement au modèle global qui génère ses propres conditions aux limites).

\section{Modélisation du régime thermique}

Les premières simulations ont porté sur le régime thermique (J. Riou,
1989), facteur déterminant des conditions d'existence, de croissance et de disparition des êtres vivants. Le modèle adopté donne, à proximité de Roscoff, une description fidèle du régime thermique, au demi-degré près, sur l'ensemble du littoral Nord-breton (fig. 11). Les résultats ont été obtenus en:

- différenciant la baie de SaintBrieuc du reste du domaine pour la détermination des coefficients de diffusion (cette baie connaissant un régime courantologique "particulier"),

- en se basant sur les bases de données EDF et IFREMER. 


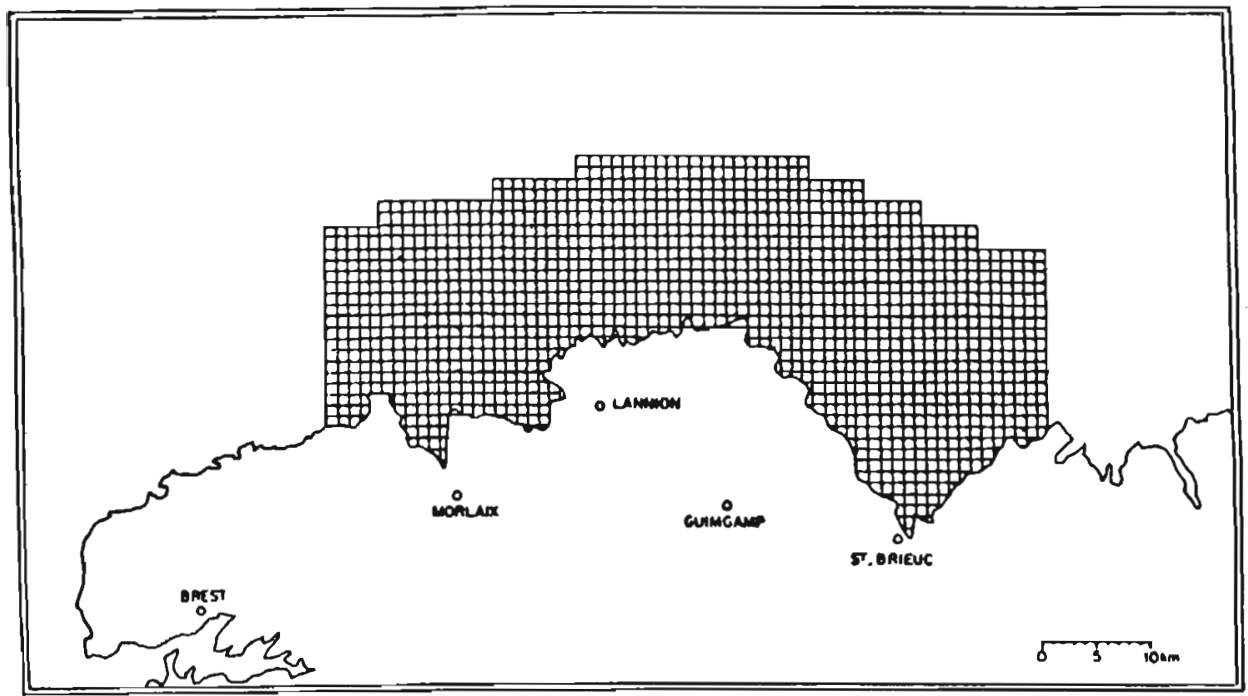

Fig. 10. Côte nord-bretonne, emprise du modèle et maillage.

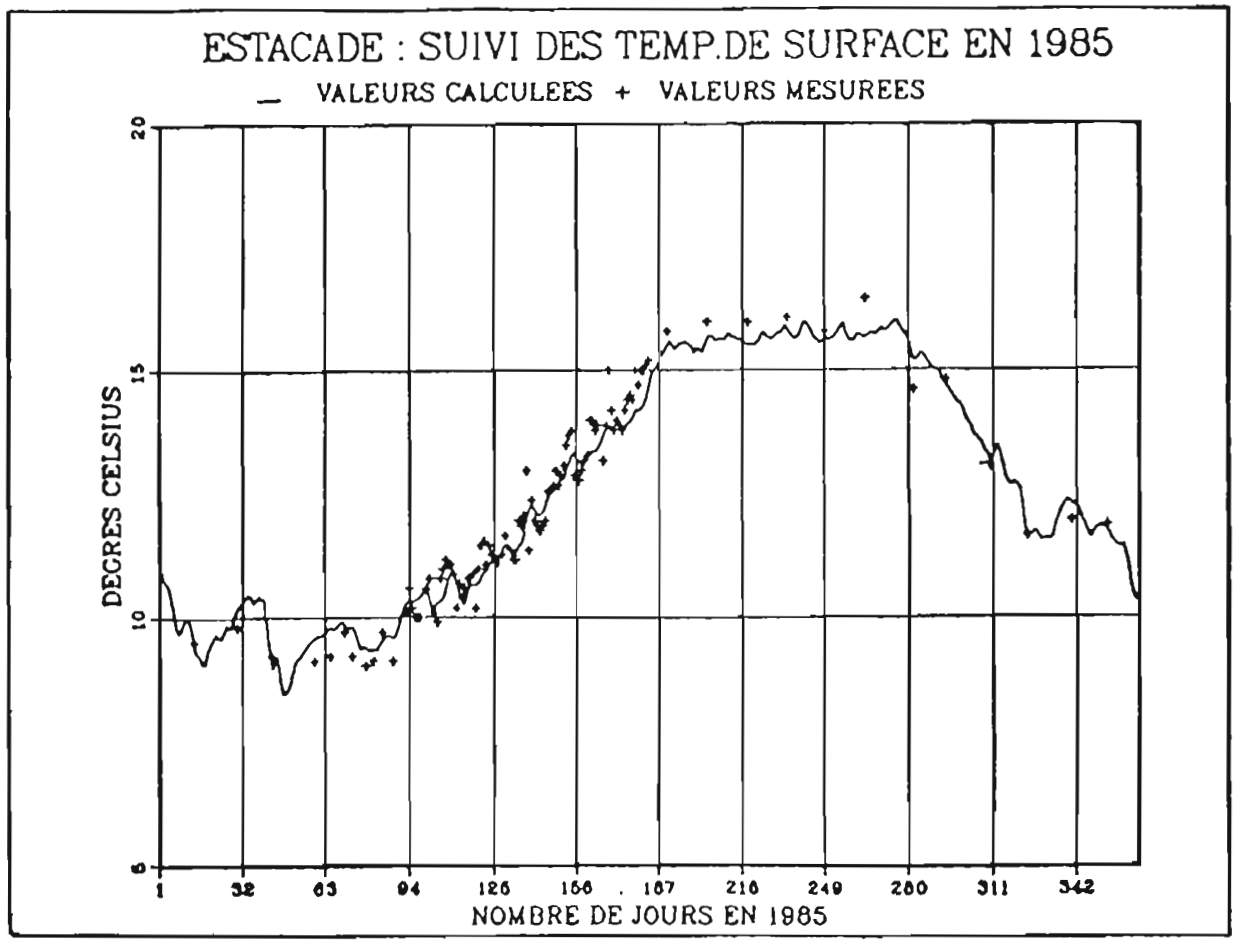

Fig. 11. Résultats du modèle thermique à l'estacade de Roscoff. 
Lumière

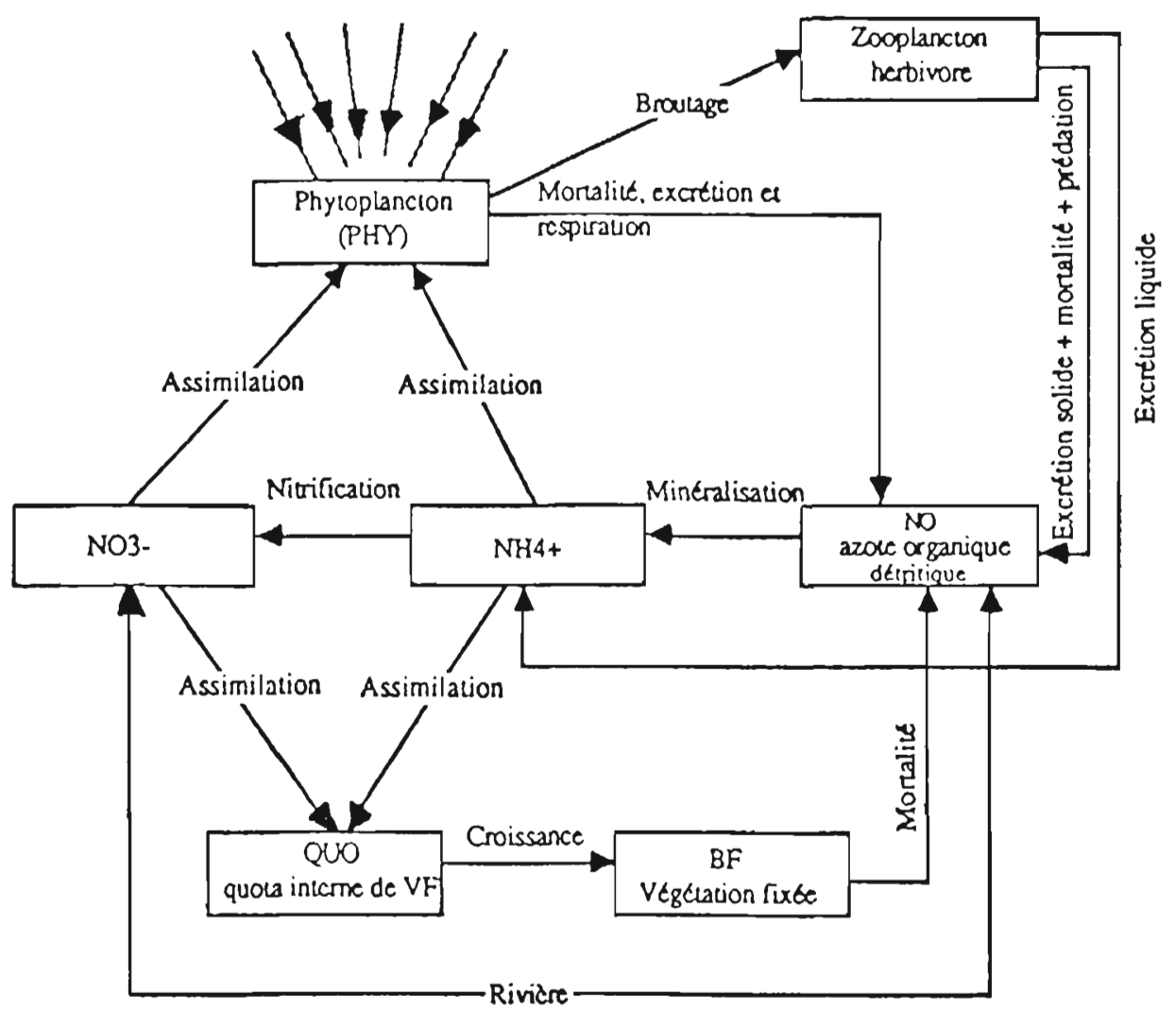

Fig. 12. Représentation schématique de l'écosystème modélisé dans le modèle cótier.

Les calculs, obtenus en conservant les mécanismes décrits dans le modèle thermique développé à l'échelle de la Manche, montrent qu'à l'échelle côtière, les échanges locaux d'énergie à l'interface eau-atmosphère et le mélange vertical et horizontal restent les facteurs essentiels du régime thermique. Le brassage hori- zontal des masses d'eau, dû aux courants de marée, joue un rôle important puisque les eaux côtières dans la région de Roscoff présentent les caractéristiques thermiques d'une masse d'eau venue du large.

\section{Première modélisation de l'écosystème}

Après ces premiers résultats satisfaisants, la démarche a consisté à tes- 


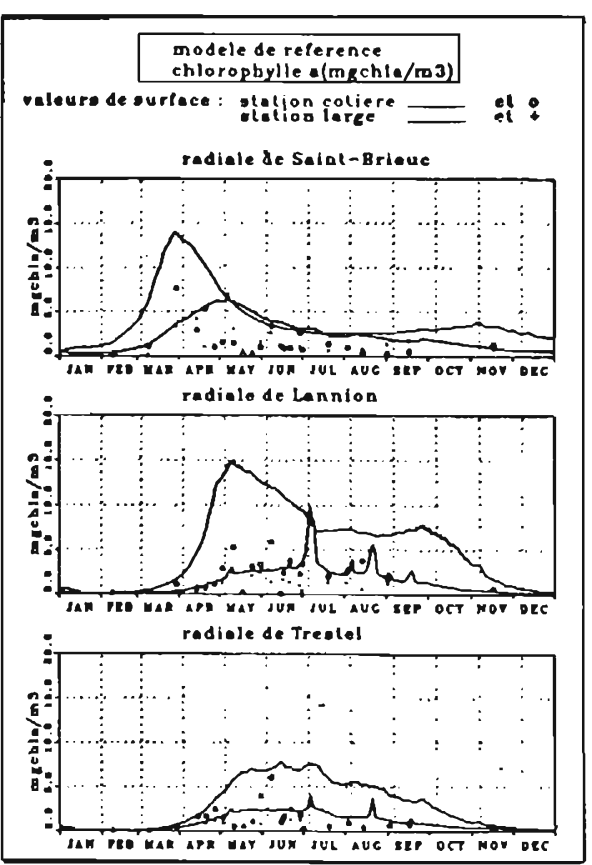

Fig. 13. Modèle de référence; comparaison calculs-mesures de la concentration en chlorophylle a en surface $\left(\mathrm{mg} \mathrm{Chla} / \mathrm{m}^{3}\right)$ aux extremités cóte-large des radiales de Saint-Brieuc, Lannion, Trestel.

ter, sur le domaine côtier, la partie biologique du modèle global.

La qualité descriptive du modèle a pu être évaluée à partir des mesures spécifiques obtenues au cours d'une campagne réalisée sur le littoral NordBreton, entre juin 1987 et juin 1988 (campagne Smart) (Riou, 1990).

L'examen des courbes simulées en quelques points de mesure, pour les variables chlorophylle a, indicateur de la biomasse phytoplanctonique et la concentration en nitrates montre la limite de représentativité du modèle (fi-

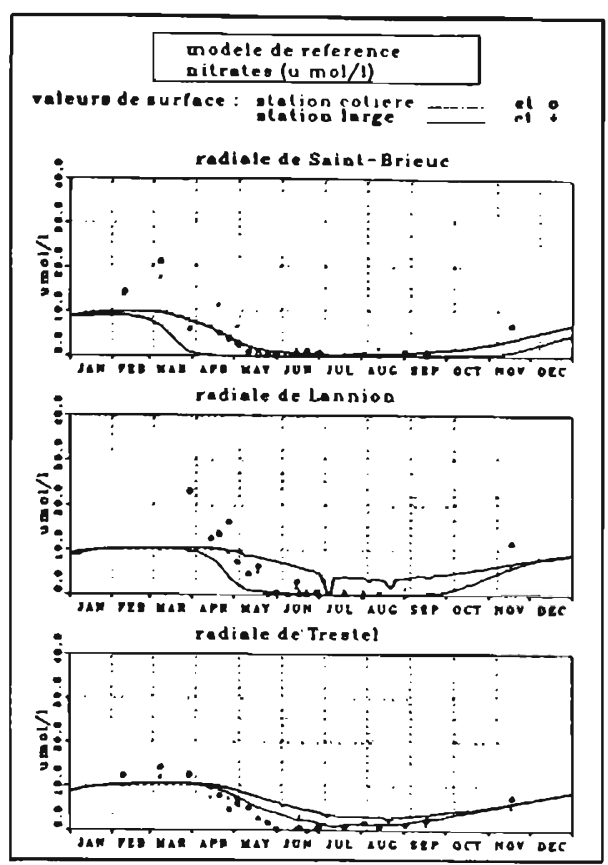

Fig. 14. Modèle de référence; comparaison calculs-mesures de la concentration en nitrate en surface $(\mu \mathrm{mol} / \mathrm{l})$ aux extrémités côte-large des radiales de Saint-Brieuc, Lannion. Trestel.

gures 13 et 14). On constate en effet des écarts entre calculs et mesures:

- les poussées phytoplanctoniques printanières sont surestimées pour l'ensemble des mailles considérées,

- les teneurs en nitrates calculées en fin d'hiver (février-mars) sont bien trop faibles,

- le modèle ne reproduit pas l'épuisement observé d'azote nitrique en été sur l'ensemble de la radiale de Trestel et sur l'extrémité large de la radiale de Lannion, alors que les deux 


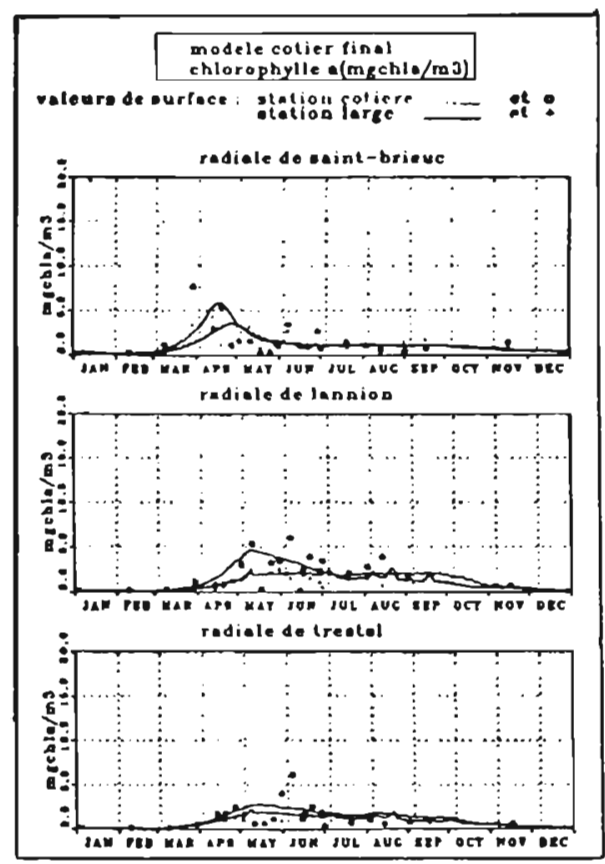

Fig. 15. Modèle phytoplanctonique côtier final; comparaison calculs-mesures de la concentration en chlorophylle a en surface $\left(\mathrm{mg} \mathrm{Chl} / \mathrm{m}^{3}\right)$ aux extrémitès cóte-large des radiales de SaintBrieuc, Lannion, Trestel.

faits précédents auraient dû favoriser cet épuisement,

- contrairement à ce qu'indiquent les mesures, il n'y a pas de parallélisme dans la chute printanière des nitrates à la côte et au large.

\section{Le modèle côtier}

Ces conclusions montrent clairement que les hypothèses adoptées dans le modèle biologique global ne suffisent

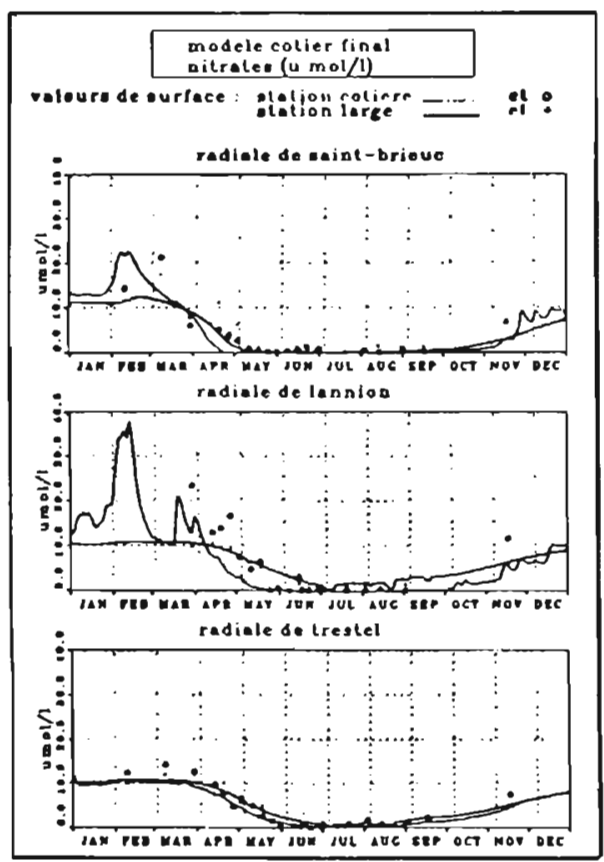

Fig. 16. Modèle phytoplanctonique côtier final; comparaison calculs-mesures de la concentration en nitrate en surface $(\mu \mathrm{mol} / /)$ aux exirémités côte-large des radiales de Saint-Brieuc, Lannion, Trestel.

pas à décrire l'évolution du phytoplancton côtier.

II apparaît que le problème principal réside dans la mauvaise représentation des processus d'apport et de disparition de l'azote nitrique. En effet, alors que ce nutrilite est sous estimé en fin d'hiver, il est clair que l'apport supplémentaire qu'il faudrait introduire pour atteindre les niveaux observés doit être plus que compensé par un puits au cours du printemps, afin de limiter, voire diminuer, la poussée phytoplanctonique printanière. 
Deux réponses ont été avancées pour résoudre ces problèmes:

a) processus d'apport d'azote en hiver:

On connaît depuis plusieurs années les problèmes de pollution en nitrates des rivières et des nappes phréatiques en Bretagne, liés à l'intensification des pratiques agricoles et l'utilisation abondante des engrais azotés: des teneurs voisines de $500 \mu \mathrm{mol}^{l^{-1}}$ sont régulièrement atteintes dès le début de l'été dans l'estuaire de Morlaix (Wafar et al., 1983) et le SRAE a estimé à 4600 tonnes de nitrates les rejets déversés en 1981 par les rivières sur la bande côtière étudiée (Assoire, 1985). Ces apports en sels nutritifs des rivières, phénomène commun à tous les fleuves côtiers, contribuent à augmenter la fertilité des systèmes côtiers, pouvant même engendrer des développements excessifs de phytoplancton ou de végétation benthique.

Ces apports sont introduits dans le modèle, à partir d'une reconstitution journalière des rejets en nitrates, en fonction du débit des rivières, pour les principaux cours d'eau du littoral (Riou, Gosse, 1990).

\section{b) processus de disparition de l'azote au printemps:}

L'originalité d'un écosystème côtier, par rapport à l'écosystème océanique en général, et la Manche en particulier, réside dans la prééminence du domaine benthique sur le domaine pélagique en ce qui concerne la production primaire. Ainsi, les eaux bretonnes se caractérisent par des grands champs de laminaires, qui représentent au moins $50 \%$ de la production primaire des fonds inférieurs à 50 mètres, $70 \%$ pour les fonds inférieurs à 12 mètres (Chassé, 1983). Le compartiment benthique se présente donc comme un compétiteur très sévère pour le phytoplancton visà-vis de la disponibilité en nutriments.

Un compartiment végétal benthique, à quota intracellulaire en azote variable, est introduit dans le modèle, sur la base des résultats obtenus par Kerambrun (1984) dans son étude des champs de laminaires en Bretagne (Riou, 1990).

Les différents processus pris en compte dans le modèle dit "côtier" sont résumés sur la figure 12. Les résultats des simulations (fig. 15 et 16) montrent une amélioration sensible:

- les poussées phytoplanctoniques printanières ont été ramenées à des valeurs raisonnables pour la région et avoisinent les $5 \mathrm{mg} \mathrm{chla} / \mathrm{m}^{3}$,

- les niveaux de chlorophylle a en période estivale sont conformes aux mesures, que ce soit à la côte ou plus au large,

- le modèle rend bien compte des variations spatiales de la distribution de la chlorophylle a, qui varie à la fois dans le sens côte-large et est-ouest (les premières poussées ont lieu à l'est du domaine étudié),

- l'épuisement en azote nitrique en période estivale est correctement représenté,

- l'introduction des rejets azotés a permis d'atteindre les fortes valeurs en nitrates mesurées en fin d'hiver sur 
tout le littoral (presque $20 \mu \mathrm{mol} . \mathrm{I}^{-1}$ à Saint-Brieuc et $40 \mu \mathrm{mol} . \mathrm{I}^{-1}$ à Lannion).

L'effet potentiel des apports azotés d'origine fluviale sur la biomasse phytoplanctonique est limité du fait de l'abondance du couvert végétal benthique, qui "pompe ", avant le démarrage des poussées phyto-planctoniques printanières, une grande partie des rejets azotés, afin de constituer des réserves intracellulaires.

\section{DU MODĖLE GLOBAL AU MODĖLE CÔTIER: PROBLÈMES DES CONDITIONS AUX LIMITES}

Les résultats du modèle global ont, rappelons-le, servi à générer les conditions aux limites en mer ouverte du modèle côtier, conditions auxquelles les résultats de ce dernier modèle se révèlent très sensibles. Or, ces conditions aux limites se situent dans la zone frontale de la Manche, zone de transition entre la partie Ouest de la Manche, aux eaux bien mélangées, et la partie Est stratifiée. La position de ce front n'est pas définie précisément dans le modèle global, mal calé faute d'une base de données suffisante. On constate en effet que le modèle global surestime, dans cette région frontale, le gradient thermique et les concentrations en nutriments dans la couche du fond. Ceci entraîne un accroissement, par les frontières du large, des réserves en nitrates du domaine côtier.

La qualité des résultats du modèle côtier, au-delà de la "réalité» des phénomènes modélisés, liés à l'échelle de description, est étroitement liée, dans cette stratégie des modèles en boîte, à la qualité des résultats du modèle global. II apparaît que l'accroissement de la "complexité " du modèle local passe avant tout par la constitution de la base de données nécessaire au calage fin du modèle Manche.

\section{CONCLUSION}

Devant la complexité des phénomènes mis en jeu dans la description de l'écosystème marin, EDF a adopté une démarche progressive, consistant à partir du simple pour aller peu à peu vers le compliqué. Cette technique, qui consiste à augmenter graduellement les difficultés, se retrouve à différents niveaux:

- au niveau des objectifs d'abord. Le modèle global a permis, dans un premier temps, de simuler le comportement moyen de l'écosystème phytoplanctonique à l'échelle de la Manche; dans un deuxième temps, l'application de cette démarche à une zone littorale a nécessité le développement d'un modèle plus spécifique, le modèle côtier.

- au niveau de l'emprise spatiale ensuite. A l'exemple des modèles dits emboîtés largement utilisés en hydrodynamique, l'emprise géographique des modèles utilisés a été réduite progressivement, de façon à avoir une image de plus en plus précise du système étudié. 


\section{- au niveau des phénomènes à} modéliser ensuite. Si l'application du schéma retenu pour décrire la Manche dans son ensemble a montré une bonne adéquation à l'échelle régionale, il est apparu que les mécanismes biologiques modélisés n'étaient pas suffisants à l'échelle locale. La structure du modèle s'est donc progressivement étoffée, tout en s'attachant, à chaque étape, à ne retenir que les mécanismes essentiels.

On dispose donc aujourd'hui d'un éventail d'outils numériques appliqués au milieu marin, permettant de mieux comprendre le fonctionnement de l'écosystème, de tester des situations types, d'étudier l'impact de rejets polluants.

\section{BIBLIOGRAPHIE}

Assoire J. 1985. Bilan en azote et en phosphore de la péninsule bretonne. DEA d'agronomie, Rennes.

Agoumi A., 1982. Modélisation du régime thermique de la Manche. Thèse de Docteur Ingénieur de l'Ecole Nationale des Ponts et Chaussées Paris.

Agoumi A., Enderle M.J. et Gras R.A., 1983. Modélisation du régime thermique de la Manche. Oceanologica acta, 6. 393-406.

Agoumi A., Gosse P. and Khalanski M., 1985. Numerical modelling of the influence of the vertical thermal structure on phytoplanktonic growth in the English channel. Proceedings of the nineteeth european marine biology symposium, Plymouth, Devon, U.K.
16-21 september 1984, Ed. P.E. Gibbs, p. 23-38.

Agoumi A., 1985. Modélisation de l'écosystème pélagique en Manche; étude de l'influence des phénomènes physiques sur le système planctonique. Thèse de doctorat d'état, université Pierre et Marie Curie, Paris $6^{\ominus}$.

Chassé C., 1983. Le potentiel de production des végétaux marins des côtes de France. Biomasse actualité, $n^{\circ}$ spécial, $\mathrm{n}^{\circ} 3$, p. 8-11.

Kerambrun L., 1984. Contribution à l'étude de la fertilité des fonds rocheux côtiers de Bretagne. Thèse de $3^{\mathrm{e}}$ cycle, université de Bretagne Occidentale, $150 \mathrm{pp}$.

Regimbaud J.F., 1987. Application de deux codes bidimensionnels en éléments finis à l'étude de l'impact thermique de centrales nucléaires. Projet de fin d'étude, Ecole Nationale des Ponts et Chaussées.

Riou J., 1989. Modélisation du régime thermique sur le littoral nord-breton. Hydroécol. Appl. 1/2, pp. 19-31.

Riou J., 1990. Modèle d'écosystème phytoplanctonique marin sur le littoral nord-breton (Manche Occidentale). Thèse de $3^{\theta}$ cycle, Institut National Polytechnique de Toulouse. $430 \mathrm{pp}$.

Riou J., Gosse P., 1990. Effet potentiel des apports d'azote d'origine fluviale sur le phytoplancton marin entre Roscoff et Saint-Brieuc. La Houille Blanche, $n^{\circ} 3 / 4$, pp. 249-256.

Wafar M., Le Corre P., Birrien J.L., 1983. Nutrients and primary production in permanently well-mixed temperate coastal waters. Estuarine and Coastal Shelf Science 17(4), pp.431-436.

Warluzel A., Manoha B., 1977. Modèle mathématique des courants de marée en Bretagne. Rapport EDF/DER E42/77 $n^{\circ} 6$. 